\title{
Intelligent multi-dose medication controller for fever: From wearable devices to remote dispensers ${ }^{\text {मr }}$
}

\author{
J. Medina*, M. Espinilla, Á.L. García-Fernández, L. Martínez \\ Department of Computer Science, University of Jaén, Spain
}

\section{A R T I C L E I N F O}

\section{Article history:}

Received 30 December 2016

Revised 11 March 2017

Accepted 13 March 2017

Available online 22 March 2017

\section{Keywords:}

Intelligent Medication Controller

Wearable data streams

Fever multi-dose analysis

Remote medication dispenser

Fuzzy logic

\begin{abstract}
A B S T R A C T
In this work, an Intelligent Medication Controller which analyzes the data streams from body temperature provided by a wearable device is proposed in order to dispense in a low-cost remote dispenser installed at home. The main innovation of our approach is a pharmacokinetic and pharmacodynamic analysis based on the successful fuzzy linguistic approach and fuzzy logic. This analysis provides accuracy and adherence to patient fever in the decision making of medication intakes, adequating the doses and waiting time based on previous intakes. In order to show its efficiency, a case study is presented, in which a complete range of fever episodes is analyzed to compare the decision making in medication intakes of antipyretics between the human expert and the proposed Intelligent Medication Controller. Our proposal has obtained an encouraging performance when recommending medication intakes in a flexible way and assuring a secure response for contraindication cases.
\end{abstract}

(C) 2017 Elsevier Ltd. All rights reserved.

\section{Introduction}

Smart objects are devices which interact with each other and cooperate with their neighbors to reach common goals [1]. The development of smart objects and environments is related to new computer paradigms, such as Internet of Things (IoT) [2], where disciplines like Ubiquitous Computing (UC) [3] have enabled driving daily activity to computer sciences by means of assisted living [4].

In this paper we have focused on modelling and expediting a critical activity: medication intakes at home, which has become a such daily activity due to the fact that half of the inhabitants of developed countries have a chronic disease [5]. It is even more decisive for elderly people, whose medication administration errors due to non-compliance with medications directions : i) account $25-40 \%$ of all medication errors, ii) are the cause of approximately $10 \%$ of hospitalizations and iii) produce $23 \%$ of hospitalizations of elderly people incomes [6]. Moreover, adverse drug reactions are an important clinical issue with a high rate prevalence in hospital stays [7] and in patient homes [8].

For these important reasons, we have focused on integrating the decision making about medication intakes by means of the innovative paradigms of AmI, UC and IoT. Our proposal describes an Intelligent Medication Controller for Fever, which

\footnotetext{
is Reviews processed and recommended for publication to the Editor-in-Chief by Guest Editor Dr. M. Karuppiah.

* Corresponding author:

E-mail addresses: jmquero@ujaen.es (J. Medina), mestevez@ujaen.es (M. Espinilla), algarcia@ujaen.es (Á.L. García-Fernández), martin@ujaen.es (L. Martínez).
} 
Table 1

Mobile applications for medication.

\begin{tabular}{|c|c|c|}
\hline Names & Features & Users \\
\hline $\begin{array}{l}\text { - Medisafe } \\
\text { - Mi Pill } \\
\text { - Dosecast }\end{array}$ & $\begin{array}{l}\text { - Reminder of intakes } \\
\text { - Reminder of replacement } \\
\text { - Report of intakes }\end{array}$ & $\begin{array}{l}\text { - Patients } \\
\text { - Relatives and caregivers } \\
\text { - Health personnel }\end{array}$ \\
\hline $\begin{array}{l}\text { - AEMPS CIMA } \\
\text { - iDoctus } \\
\text { - Assist UK: Drug Interactions } \\
\text { - Medication Guide }\end{array}$ & $\begin{array}{l}\text { - Database of authorized medications (for each country) } \\
\text { - Description, indications, precautions and contraindications and adverse reactions } \\
\text { - Pill identifier }\end{array}$ & $\begin{array}{l}\text { - Patients } \\
\text { - Pharmacists } \\
\text { - Health personnel }\end{array}$ \\
\hline $\begin{array}{l}\text { - PK Curve } \\
\text { - T Calc }\end{array}$ & - Calculator of function of bioavailability & $\begin{array}{l}\text { - Students } \\
\text { - Industry scientists } \\
\text { - Pharmacists } \\
\text { - Health personnel }\end{array}$ \\
\hline
\end{tabular}

analyzes the data streams from wearable body temperature devices and expends adequate medication in a remote dispenser installed at home.

The remainder of the paper is structured as follows: in Section 2 we present related works, in Section 3 the approach and their components are described in detail, in Section 4 an evaluation from a case study is discussed. Finally, in Section 5 conclusions and future works are pointed out.

\section{Related works}

In this section, we describe previous approaches and works which are closely related to the functionality of the Intelligent Medication Controller proposed in this paper.

On previous approaches on medication control, we can find three relevant works for monitoring the medication intakes of a patient [9-11] related to our approach:

- iMat: Intelligent medication administration tool [9]. It controls the medication intakes using crisp interval time restrictions and maximum dose of medications. However, non adherence to a user conditions or symptoms, such as fever or pain, is related with the time and dose of medication proposed.

- A remote monitoring system of medication compliance using distributed RFID readers in an intelligent living environment [10]. It is a fuzzy oriented approach in which the compatibility of medication intakes is related to the interaction with other objects in a marked world, which can be incompatible with medication.

- Drug identification and interaction checker based on IoT to minimize adverse drug reactions and improve drug compliance [11]. In this work, a drug identification by near contact technologies (RFID, NFC or barcodes) provides a verification of drug compliance to calculate possible adverse interactions. Adherence to symptoms is not analyzed in order to adequate medication doses and medication effect in times.

In addition to the academic works, several mobile applications has facilitated prescribing, reminding and dispensing drugs. They can be grouped into two main categories: reminders for medication intakes and databases of medications (vademecum). On the calculus of bioavailability of medication, scarce applications have been developed. We show an example of mobile applications for handling medications in Table 1.

Previous works have focused on reminding medication pills, checking compatibilities between medications or ensuring rigid waiting time for next intakes. However, the isolated information of medication intakes and its adverse reactions is not enough to develop flexible systems which recommend the doses and waiting time of medication based on the evolution of a symptom. For example, an Intelligent Medication Controller for fever would answer questions such as how much paracetamol is suggested for my persistent high fever if I took $500 \mathrm{mg}$ five hours ago?. This question is the relevant aim of our proposed Intelligent Medication Controller: providing accuracy and adherence in the decision making of medication intakes based on the evolution of the patient fever.

The Intelligent Medication Controller would provide an optimal adherence of medication to fever based, giving answer to spacing the medication intakes based on the fever evaluation and minimizing medication intakes. It could be deployed for risk-free everyday diseases, where a real time monitoring by doctors at home is non-viable in health systems. However, we note that previously to enable the Intelligent Medication Controller, a medical prescription of medication based on a medical diagnosis is recommended, as well as, the periodic review of the patient by health personnel.

In order to deal with this issue, on the first hand, we propose a intelligent medication analysis based on a fuzzy linguistic approach and fuzzy logic [12]. In addition, we have included fuzzy temporal processing of sensor streams, which allow us to represent temporal knowledge in an intuitive and flexible way in several contexts [13].

Moreover, we describe a solution based on processing data streams from wearable devices because of becoming facilitators of health behavior [14]. In general way, mHealth facilitates the adherence to chronic diseases management [15]. 


\section{Intelligent medication controller for fever}

In this section, we present an Intelligent Medication Controller for Fever which controls the presence of fever in patients collected by wearable body temperature devices, analyzing the pharmacokinetic [16] and pharmacodynamic [17] of multidose medication intakes in time. On the first hand, the pharmacokinetic analysis is calculated using the bioavailability of a medication, which measures the amount of administered dose absorbed into the systemic circulation. It is determined by a function of time which estimates the plasma concentration $(\mathrm{mg} / \mathrm{l})$ based on a previous medications intakes. On the second hand, the pharmacodynamic analysis relates the concentration of medication to other measurements, such as efficacy with respect to a symptom, also known as intrinsic activity [18]. Moreover, we include a toxicokinetic analysis [19], which attends to control basic principle: sola dosis facit venenum. For that, we evaluate the limits of concentration levels of the possible doses preventing the occurrence of contraindications.

For estimating the presence of fever, the Intelligent Medication Controller analyzes the temperature data from wearable devices applying a previously developed approach [20], in which a methodology based on linguistic fuzzy method is proposed. Evaluating the degrees of fever and efficacy of medication under a fuzzy logic approach, the Intelligent Medication Controller is able to recommend the most adequate doses and medications. Finally, when the patient chooses taking a compatible medication, the Intelligent Medication Controller connects to a remote medication machine to dispense the dose.

The Intelligent Medication Controller includes a Component-Based Architecture, where the development of the fields described previously has been evolved with next modules:

- 1) Estimating the bioavailability. Based on the previous medication intakes, a transformation from the discrete domain of medication intakes to a continuous domain of plasma concentration over time is proposed. Its aim is increasing the accuracy in determining the degree of presence of medication over time.

- 2) Inferring efficacy and contraindication of medication intakes. In the field of pharmacology, the medication effect is defined as a functional response in the face of symptoms [21]. Here, we apply a fuzzy logic processing method driven by expert knowledge to estimate the dose - response relationship from the measurement of bioavailability to a degree of medication efficacy.

- 3) Evaluating the degree of symptoms. The data from wearable devices, which monitor the body temperature, is collected in a remote database of biosignals of patients from which the Intelligent Medication Controller obtains the data streams of temperature.

- 4) Evaluating the gain in medication intakes. Based on the degree of efficacy of medication and the degree of fever, the Intelligent Medication Controller firstly analyzes the gain in taking hypothetical medications and secondly proposes and rates the most adequate. More specifically, the gain has been estimated by maximizing the adherence and minimizing the medication toxicokinetics.

- 5) Remote medication dispensing. Despite the fact that the Intelligent Medication Controller could be integrated into any medication dispenser, we present a low-cost prototype built with a 3D printer and using wireless communication.

The modules described above, which integrated as different components in the Intelligent Medication Controller for Fever, are shown in Fig. 1 and described in the following sections.

\subsection{Calculating bioavailability from medication intakes}

This subsection describes in detail how the bioavailability of a medication is computed from data of previous intakes. Previous intakes of a medication $i$ of a quantitative dose $d$ are defined as a set of timestamps of intakes: $m^{i}(d)=\left\{t_{1}^{i}, \ldots, t_{n}^{i}\right\}$

In order to provide more information to the Intelligent Medication Controller in addition to medication intakes described by their timestamps, we include a pharmacokinetic analysis computed by means of bioavailability using well-known functions of plasma concentration in time [16]. The bioavailability will add precision and accuracy in the calculation of the degree of presence of the medication.

The calculus of bioavailability is based on the functions of bioavailability for each medication dose, which are provided by the pharmaceutical labs in their development processes. The function of bioavailability $B^{i}(d, \Delta t)$ of a medication $i$ enable us to calculate the concentration in the systemic circulation $(\mathrm{mg} / \mathrm{l})$ based on the medications intakes $m^{i}(d)$, where $d$ is the dose and $\Delta t$ the time elapsed between a previous timestamp of intake $t_{j}^{i}$ and the current time $t^{*}$. By means of this function we can obtain the bioavailability from all intakes $b^{i}(t)$ of the medication $i$ at a particular point of time $t$ :

$$
\begin{aligned}
b^{i}(t) & =\sum_{i=1}^{n} B^{i}\left(d, \Delta t_{i}\right), \\
m^{i}(d) & =\left\{t_{1}^{i}, \ldots, t_{n}^{i}\right\}, \\
\Delta t_{i} & =t^{*}-t_{j}^{i}
\end{aligned}
$$

An example of the calculation of bioavailability, based on functions of bioavailability from oral medication intakes of paracetamol [22] and ibuprofen [23], is shown in Fig. 2. 


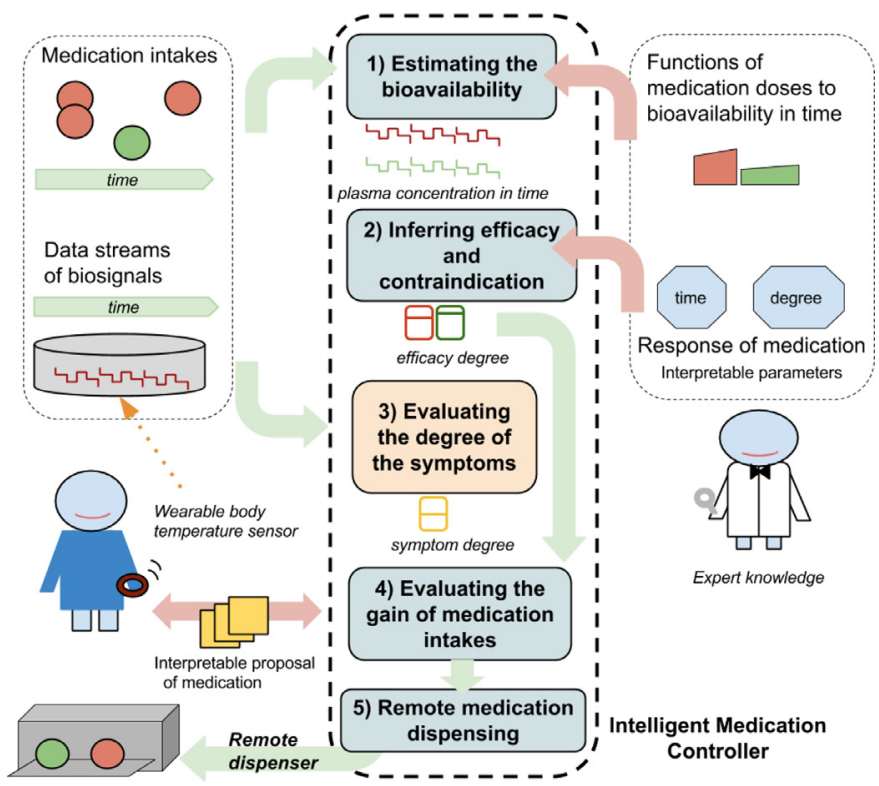

Fig. 1. Architecture of components, in which the data from patients is processed using expert knowledge to propose compatible medication intakes which are provided by a remote dispenser.

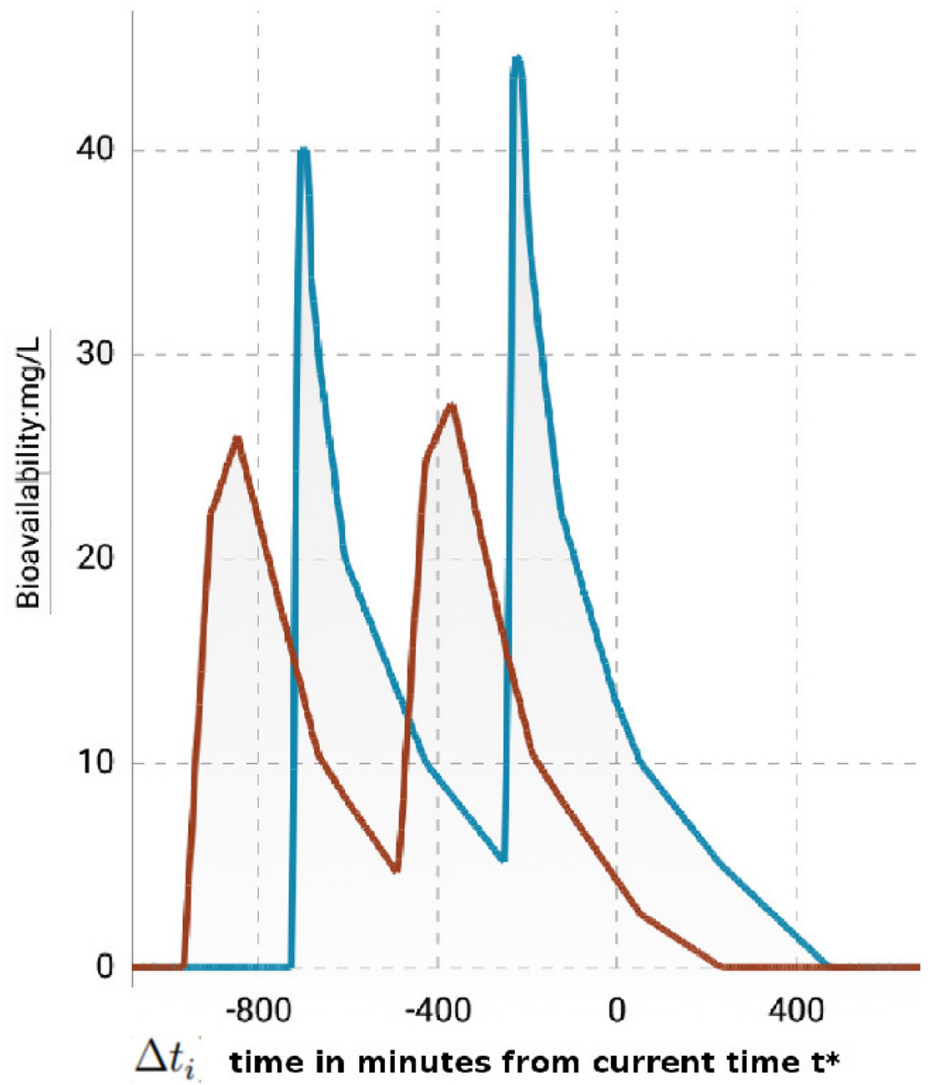

Fig. 2. Calculus of bioavailability $(\mathrm{mg} / \mathrm{l})$ for ibuprofen and paracetamol for $t^{*}=24: 00 \mathrm{~h}, \quad m^{\text {ibuprofen }}(600 \mathrm{mg})=\{16: 00 \mathrm{~h}, 08: 00 \mathrm{~h}\} \quad$ (in blue line), $m^{\text {paractamol }}(1000 \mathrm{mg})=\{20: 00 \mathrm{~h}, 12: 00 \mathrm{~h}\}$ (in red line). (For interpretation of the references to colour in this figure legend, the reader is referred to the web version of this article.) 


\subsection{Inferring efficacy from medication bioavailability and human expert knowledge}

Despite the pharmacokinetic analysis, the measurement of bioavailability is not enough to describe the direct relationship between medication intakes and a functional response. In order to obtain this information, it is necessary to integrate a pharmacodynamic analysis [17], which relates the concentration of medication to other measurements, such as efficacy, also known as intrinsic activity [18], potency or affinity of a medication with respect to a symptom. However, measuring and estimating the functional responses is a complex process due to the need for qualitative and quantitative evaluation of physiological, biochemical or psychological aspects [24]. At this point, in order to reduce the complex integration of pharmacodynamic analysis, a fuzzy logic processing method driven by expert knowledge is proposed to integrate straightforwardly the human experience and observation on calculating the degree of efficacy in medications.

Therefore, in this subsection we present how to deduce the degree of efficacy of a medication from its bioavailability concentration using expert knowledge. We have focused on efficacy due to the straightforward relation between its measurement as positive or negative percentage of response in the receptor system and the membership degree in a fuzzy set. The definition of efficacy is related to the ability of a medication to produce an expected maximum functional response [25]. Based on this concept, we include the knowledge provided by human experts in order to model the degree of efficacy of a medication. In this work, two parameters are proposed to model the membership degree of the fuzzy set of efficacy from bioavailability:

- Maximal efficacy degree of response for a medication dose $m_{d}$ to symptom $s$, defined as $V^{*}\left(m_{d}\right.$, $\left.s\right)$. This value represents the maximum degree $\in[0,1]$ of medication efficacy for responding to a symptom over the time. For example, ibuprofen $(600 \mathrm{mg})$ and paracetamol $(1000 \mathrm{mg})$ can be represented by providing best maximal response degree to fever: $V^{*}\left(\right.$ ibuprofen $_{600 \mathrm{mg}}$, fever $)=1.0, V^{*}\left(\right.$ paracetamol $_{1000 \mathrm{mg}}$, fever $)=1.0$; whilst paracetamol $(500 \mathrm{mg})$ could be evaluated with a lower value: $V^{*}\left(\right.$ paracetamol $_{500 \mathrm{mg}}$, fever $)=0.7$.

- Duration of efficacy, defined as $T^{*}\left(m_{d}, s\right)$, of a medication dose $m_{d}$ for a symptom $s$ is a measurement related to the period of time which the medication is effective [26]. It is evaluated by using expert knowledge too, which is represented as a temporal linguistic term whose temporal membership function $\mu_{T^{*}\left(m_{d}, s, \Delta t_{j}\right)}$ is defined by the time interval $\Delta t_{i}=$ $t^{*}-t_{i}, t^{*}>t_{i}$ from the current time $t^{*}$ to a previous time $t_{i}$. We propose modeling the temporal membership function by means of a well-known trapezoidal membership function $T S$ :

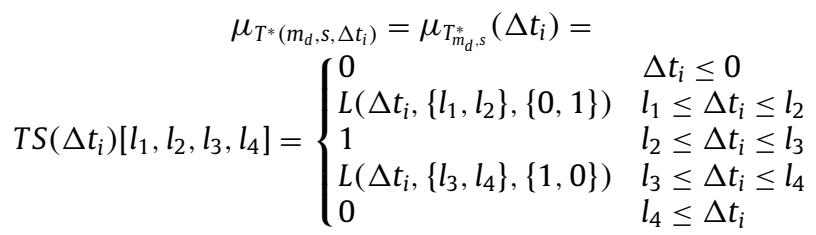

where $L\left(x,\left\{x_{0}, y_{0}\right\},\left\{x_{1}, y_{1}\right\}\right)$ is a linear approximation function of $x$ between the control points $\left\{x_{0}, y_{0}\right\},\left\{x_{1}, y_{1}\right\}$. For example, the duration of ibuprofen $_{600 \mathrm{mg}}$ could be evaluated between 4 or 6 hours, which could be defined by means of the trapezoidal membership function $T^{*}\left(\right.$ ibuprofen $_{600 \mathrm{mg}}$, fever $)=T S\left(\Delta t_{i}\right)[0,0,4 h, 6 h]$.

A perceptive reader can note that expert knowledge does not integrate bioavailability in the parameters of efficacy. This is due to the fact that bioavailability is not an observable phenomenon collectible by human experience; however, the maximal degree of response and the duration of efficacy are. In order to deduce the efficacy degree from the bioavailability, following we present how calculating the degree of medication efficacy based on the value of bioavailability from previous intakes.

Firstly, in order to operate with the bioavailability of a medications $b_{m}\left(t_{j}\right), t<t_{0}$ in a fuzzy representation, we have defined the membership function of the fuzzy set presence of bioavailability using a linear normalization which sets the maximal value of concentration to the maximal degree of presence 1, which is defined by the linear approximation function:

$$
\begin{gathered}
B_{m}(t):\left[0,\left(b^{m}(t)^{*}\right)\right] \times[0,1] \rightarrow \\
\left.B_{m}(t)=L\left(b^{m}(t),\left\{0, b^{m}(t)^{*}\right\},\{0,1\}\right), b^{m}(t)^{*}=\sup \left(b^{m}(t)\right)\right]
\end{gathered}
$$

Secondly, we calculate an aggregation degree of bioavailability in the fuzzy temporal set defined by the temporal fuzzy set duration of efficacy $T^{*}(m, s)$ for the medication $m$ and the symptom $s$ at a certain point of time $t$ using the fuzzy weighted average, which is defined as a suitable fuzzy aggregation in temporal data [13].

$$
B_{T}(m, s, t)=B_{m}(t) \cup T^{*}\left(m_{d}, s\right)=\frac{1}{\sum T^{*}\left(m_{d}, s\right)} \sum_{t<t^{*}} B_{m}(t) \times T^{*}\left(m_{d}, s\right)
$$

$B_{T}(m, s, t)$, whose range is $\in[0,1]$, represents the presence degree of bioavailability in the temporal term defined by $T^{*}\left(m_{d}, s\right)$. In addition, we define $B_{T}\left(m_{d}, s\right)^{*}$ as the maximal value of presence degree of bioavailability in the time interval of the duration of efficacy: $B_{T}(m, s)^{*}=\sup \left(B_{T}(m, s, t)\right), \forall t \in[0, \infty]$.

Thirdly, we apply fuzzy quantifier $Q:[0,1] \times[0,1]$ to relate the presence degree of bioavailability to the efficacy degree of response $e(m, s, t)$ for the medication $m$ and the symptom $s$ at a certain point of time $t$. For that purpose, the maximal efficacy degree of response $V^{*}\left(m_{d}, s\right)$, defined by experts for certain dose of medication $m_{d}$ and symptom $s$, is used as control points in order to calculate degrees of efficacy in the complete universe of discourse (see Fig. 3 ). 


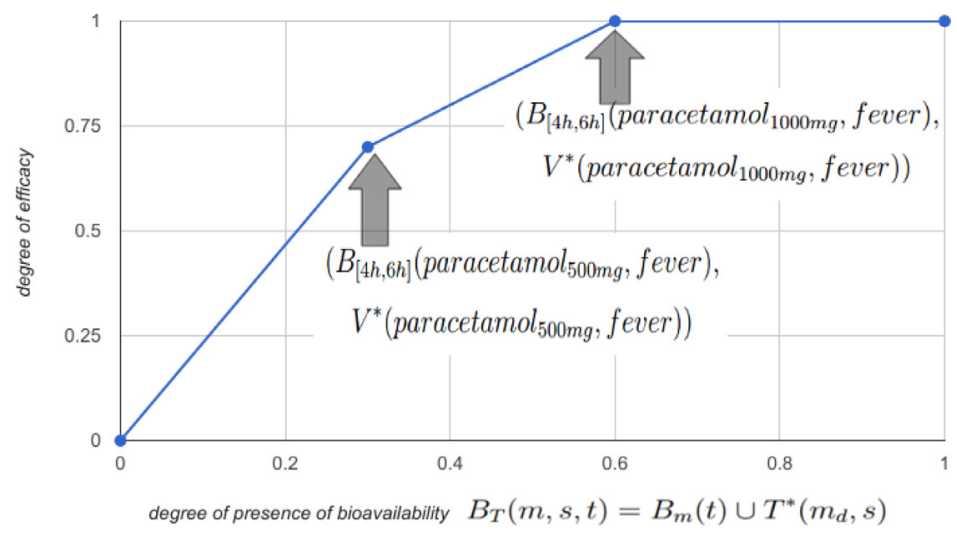

Fig. 3. Fuzzy quantification from presence degree of bioavailability to obtain the efficacy degree of medication using maximal response degrees as control points. In this example, maximal efficacy degree of response for doses of paracetamol $V^{*}\left(\right.$ paracetamol $_{500 \mathrm{mg}}$, fever $)=0.7, V^{*}\left(\right.$ paracetamol $_{1000 \mathrm{mg}}$, fever $)=1.0$, are considered, and duration of efficacy: T paracetamol, fever* $=[4 h, 6 h]$.

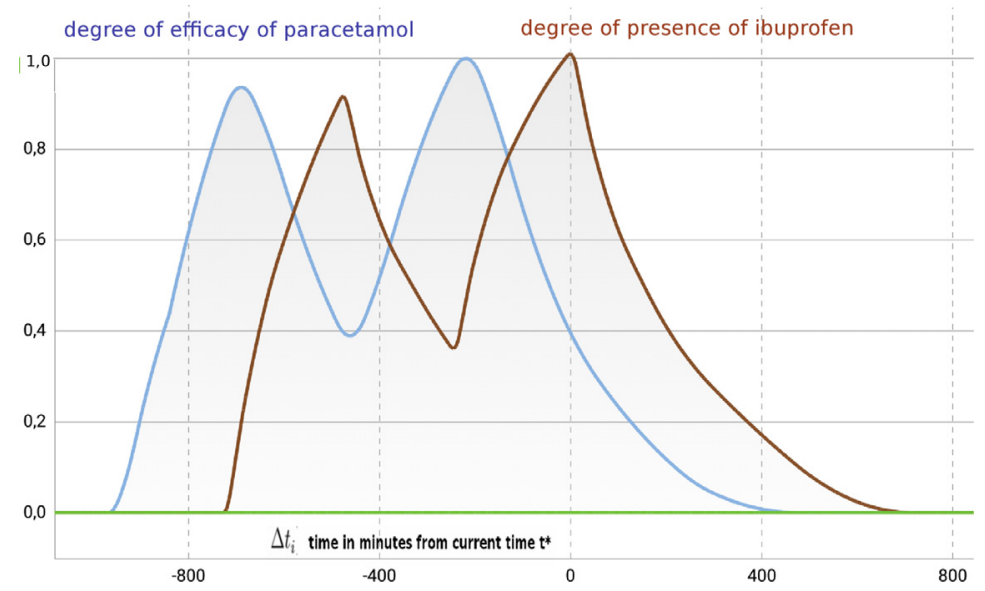

Fig. 4. Example of calculus of efficacy degree of paracetamol and ibuprofen for fever $t^{*}=24: 00 \mathrm{~h}$, $\mathrm{m}^{\text {ibuprofen }}(600 \mathrm{mg})=\{16: 00 \mathrm{~h}, 08: 00 \mathrm{~h}\}$ (in blue line), $m^{\text {paracetamol }}(1000 \mathrm{mg})=\{20: 00 \mathrm{~h}, 12: 00 \mathrm{~h}\}$ (in red line). (For interpretation of the references to colour in this figure legend, the reader is referred to the web version of this article.)

We propose the linear approximation $L\left(x,\left\{x_{i}, y_{i}\right\},\left\{x_{i+1}, y_{i+1}\right\}\right)$ between the control points of maximal efficacy degree defined by experts, to estimate the efficacy degree of response.

$$
\begin{aligned}
e(m, s, t) & =Q\left(B_{T}\left(m_{d}, s, t\right)^{*}\right)=L\left(x,\left\{x_{i}, y_{i}\right\},\left\{x_{i+1}, y_{i+1}\right\}\right), \\
x & =B_{T}\left(m_{d}, s, t\right), x \in\left[x_{i}, x_{i+1}\right], x_{i}=B_{T}\left(m_{d}, s\right)^{*}, x_{i+1}=B_{T}\left(m_{d+1}, s\right)^{*}, \\
y_{i} & =V^{*}\left(m_{d}, s\right), y_{i+1}=V^{*}\left(m_{d+1}, s\right)
\end{aligned}
$$

Finally, the value $e(m, s, t)$ represents the membership degree to the fuzzy set efficacy of the medication intakes $m$ for a symptom $s$ at a certain point of time $t$. From this value, we can define $e(m, s)^{*}$ as the maximal efficacy degree for a medication intake in a time interval: $e(m, s)^{*}=\sup (e(m, s, t)), \forall t \in[0, \infty]$.

An example of evolution of the efficacy degree over a time interval for the symptom fever, with the medication intakes of Fig. 2 and the example parameters in this section, is shown in Fig. 4.

\subsection{Inferring the contraindication degree from medication bioavailability and human expert knowledge}

In the same way as calculating efficacy degree, the contraindications derived by toxicokinetic of a set of hypothetical medication intakes must be evaluated. The method is based on integrating quantification control points for calculating the contraindication degree from the efficacy degree.

In order to calculate the degree of contraindication, we propose the use of human expert knowledge to define the fuzzy set of contraindications of medication intakes, noted by $c(m, s, t)$. This set defines the degree of contraindications. For defining the membership functions of contraindication for each medication in an interpretable way, we propose calculating them using the knowledge from experts and laboratories, which is defined by waiting time between two consecutive intakes. For 


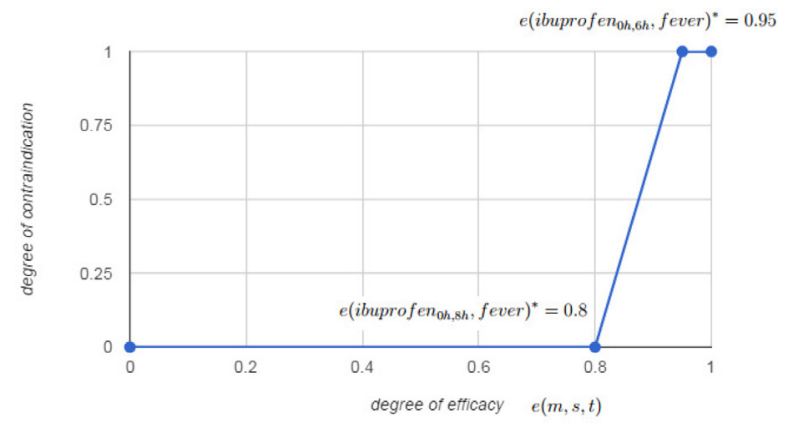

Fig. 5. Fuzzy quantification from the presence degree of bioavailability to the contraindication degree of medication using maximal efficacy degrees and waiting interval between two consecutive intakes as control points. In this example, wait interval inter intakes for ibuprofen is set to: $m_{i b u p r o f e n}\left[t_{0}^{-}, t_{n}^{-}\right]=$ $[6 h, 8 h]$.

example, a dose of ibuprofen $_{600 \mathrm{mg}}$ is suggested at most between two points of time: $m_{d}\left[t_{0}^{-}, t_{n}^{-}\right]=[6 h, 8 h]$, where less than 6 hours is intolerable (degree of contraindication 1) and more than 8h does not present any contraindication (degree of contraindication 0 ).

In order to relate the presence degree of bioavailability to the contraindication degree in the complete universe of discourse, we apply a fuzzy quantifier $[27] Q:[0,1] \times[0,1]$ defined by two control points which relates the maximal efficacy degree of medication intakes for each point of time $m_{d}=\left\{t^{*}, t^{*}+t_{0}^{-}\right\}, m_{d+1}=\left\{t^{*}, t^{*}+t_{n}^{-}\right\}$to the degrees of contraindication $\{0,1\}$ respectively.

$$
\begin{aligned}
c(m, s, t) & =Q\left(B_{T}(m, s, t)^{*}\right)=L\left(x,\left\{x_{0}, 0\right\},\left\{x_{1}, 1\right\}\right), \\
x_{0} & =e\left(m_{d}^{0}, s, t\right)^{*} ; x_{1}=e\left(m_{d}^{1}, s, t\right)^{*}, \\
m_{d}^{0} & =\left\{t^{*}, t^{*}+t_{0}^{-}\right\} ; m_{d}^{1}=\left\{t^{*}, t^{*}+t_{n}^{-}\right\}
\end{aligned}
$$

Finally, the value $c(m, s, t)$ represents the membership degree to the fuzzy set contraindication for a medication intakes $m$ to a symptom s at a point of time $t$. An example with ibuprofen $(600 \mathrm{mg})$ and waiting time between two consecutive intakes $m_{d}\left[t_{0}^{-}, t_{n}^{-}\right]=[6 h, 8 h]$ is shown in Fig. 5.

\subsection{Evaluating the degree of symptoms}

Evaluating the degree of symptoms in patients is a research field which has been widely studied in many nursing and medical contexts. It also includes the analysis of physiological, psychological, sociological and spiritual information [24]. For example, evaluating subjective symptoms, such as pain [28], is gathered with assessments of indicators, which have been analyzed by nursing theory proposed in a qualitative or quantitative [29] way. Other recent methodologies for wearable sensor-based systems describe real-time analysis for monitoring and prognosis [30]. Both health assessments from human experts and data streams from wearable sensors can be translated into fuzzy models [20] enabling the evaluation of medication taking based on the symptoms of the patients. In this work, we are focused on detecting the presence of fever by analyzing data from wearable body temperature sensors.

The degree of presence of fever is calculated using the approach of a previous work [20], in which a methodology for real-time linguistic analysis of data streams generated from wearable e-health devices is proposed. The main advantage of this proposal is including fuzzy temporal terms and fuzzy quantifiers defined by expert knowledge, to monitorize the fever of patients. This previous work is the base to compute the degree of fever of the patient, which enables calculating the gain in medication efficacy.

\subsection{Calculating adherence of medication efficacy to symptom}

In this subsection, we propose a methodology to compute the degree of gain for hypothetical medication taking to response to a degree of presence of a symptom.

Firstly, as we detailed previously, the degree of presence of fever is calculated using the methodology of a previous work [20], in which a linguistic analysis of data streams from wearable body temperature devices is proposed. This work enables us to calculate the gain in medication efficacy from the degree of presence of fever at a given current time.

Secondly, based on the degree of presence of fever $s\left(t^{*}\right)$ in the current time $t^{*}$, the Intelligent Medication Controller aims evaluating a new hypothetical medication intake $m$ in $t^{*}$. For that purpose, we compare and aggregate the degree of medication efficacy versus the symptom degree in a future temporal window $\left[t^{*}, t_{f}\right]$ from the current time $t^{*}$ to obtain the prospective degree of adherence $a\left(m, s, t^{*}\right)$ :

$$
a\left(m, s, t^{*}\right)=\bigcup_{t}^{\left[t^{*}, t_{f}\right]}\left\|s\left(t^{*}\right)-e(m, s, t)\right\|
$$




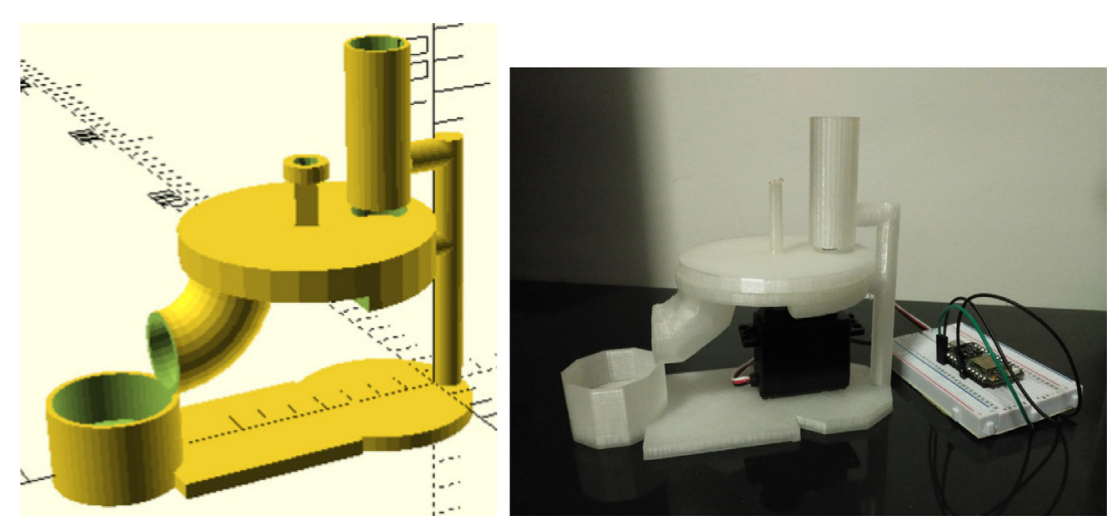

Fig. 6. 3D design and a picture of the prototype of remote medication dispense.

In the same way, the degree of contraindication is aggregated in the future temporal window [ $\left.t^{*}, t_{f}\right]$ starting from the current time $t^{*}$ to obtain the prospective degree of contraindication $a\left(m, s, t^{*}\right)$ :

$$
c\left(m, s, t^{*}\right)=\bigcup_{t}^{\left[t_{0}, t_{f}\right]} c(m, s, t)
$$

Finally, the degree of gain $g\left(m, s, t^{*}\right)$ of a hypothetical medication intake $m$ is used as matching degree for the fuzzy rule:

IF adherence of medication to fever $a\left(m, s, t^{*}\right)$ AND NOT contraindication of medication $c\left(m, s, t^{*}\right)$ THEN recommending medication with degree $g\left(m, s, t^{*}\right)$ in which the recommendation degree is computed based on the fuzzy sets of adherence and not contraindication and the fuzzy operators:

$$
g\left(m, s, t^{*}\right)=a\left(m, s, t^{*}\right) \cap \neg c\left(m, s, t^{*}\right)
$$

Based on the fuzzy rule and operations, we note that:

- The contraindication of several medications can be aggregated concatenating several antecedents in the shape of $A N D$ NOT contraindication of medication $c\left(m_{2}, s, t^{*}\right)$.

- Diverse fuzzy operators can be defined to provide different semantic when aggregating in the temporal window or when comparing the similarity between the degrees of medication efficacy and the degrees of symptom.

\subsection{Prototype of remote medication dispenser}

In this section, a low-cost prototype of a smart medication dispenser integrated at home is presented. The key points of the prototype are:

- It features a 3D printable physical structure under a Creative Common License ${ }^{1}$, in order to enable developers to build, print and design their own medication dispensers. It has been developed with OpenScad ${ }^{2}$, an open source tool for CAD.

- Integration with a remote service which enables the medication dispensing. For this purpose, a smart board for IoT is integrated: Photon Particle ${ }^{3}$, which includes a Wi-Fi chip to deploy services on the connection oriented protocol IEEE 802.11. The smart board is connected to a $5 \mathrm{~V}$ servomotor which produces the rotation of the two wheels of the structure.

In Fig. 6, we show the 3D design and a picture of the prototype of remote medication dispenser. A version is installed in the Smart Lab located in the Center for Advanced Studies in Information and Communication Technologies in the University of Jaen ${ }^{4}$. Finally, the code of OpenScad and the remote services for Photon Particle are available in next website ${ }^{5}$.

\section{Evaluation of the Intelligent Medication Controller for fever}

In this section we present the results related to the evaluation of the Intelligent Medication Controller in hypothetical fever situations monitored by a wearable body temperature device. The case study has been designed to analyze the ability of the controller to suggest adequate medications which are expended by the remote medication dispenser from the body

\footnotetext{
1 https://creativecommons.org/.

2 http://www.openscad.org/.

3 https://www.particle.io/.

4 http://ceatic.ujaen.es/es/smartlab.

5 http://sinbad2.ujaen.es:8068/medication/medication.html.
} 
Table 2

The columns are related in order to: i) medication and dose, ii) averaged value of maximum effectiveness completed by experts, iii) scaled and rounded maximal efficacy degree, iv) values completed by experts of duration regarding efficacy in hours from medication intake (value and frequency) and v) proposed trapezoidal membership function.

\begin{tabular}{lllll}
\hline Medication and dose & Effectiveness [0,10] & $V^{*}\left(m_{d}, s\right)$ & Duration of efficacy (in hours) & $\mu_{T^{*}\left(m_{d}, s, \Delta t_{i}\right) .}$ \\
\hline Ibuprofen (600 mg) & 8.4 & 0.8 & $3 h(3), 4 h(3), 5 h(2), 6 h(2)$ & $T S(0 h, 0 h, 4 h, 6 h)$ \\
Paracetamol $(500 \mathrm{mg})$ & 6.9 & 0.7 & $3 h(2), 4 h(6), 5 h(1), 6 h(1)$ & $T S(0 h, 0 h, 4 h, 6 h)$ \\
Paracetamol (1000 mg) & 9.6 & 1.0 & $4 h(5), 5 h(3), 6 h(2)$ & $T S(0 h, 0 h, 4 h, 6 h)$ \\
\hline
\end{tabular}

Table 3

Cases presented in the second survey.

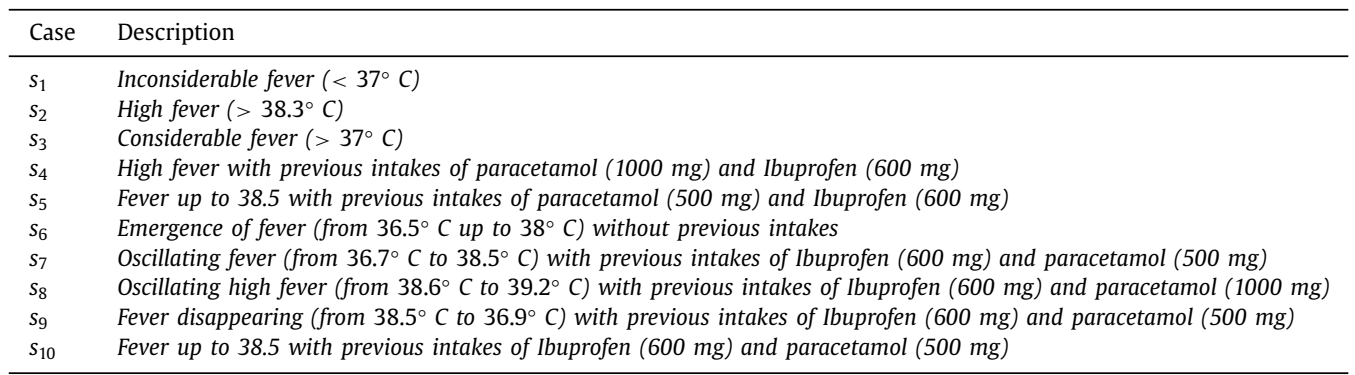

temperature of a wearable device. Here, we highlight that the use of wearable e-health devices enables more accurate in the analysis of fever degree using high rate sensor streams instead of traditional hand measurements.

The evaluation is focused on comparing the decision making regarding medication intakes between human experts and the Intelligent Medication Controller for several antipyretics, ibuprofen $(600 \mathrm{mg})$ and paracetamol (500mg and $1000 \mathrm{mg})$. For this purpose, we designed a case study with two surveys which were completed by 10 medical experts ( 2 family doctors, 3 nurses and 5 pharmacists) not related to the project.

\subsection{Considerations of the case study}

The case study was defined to evaluate the intake of antipyretics for a health adult whose weight is $80 \mathrm{~kg}$. Therefore, the aim of this work is to analyze the adherence of the Intelligent Medication Controller to expert decision making regarding two antipyretics at different doses: paracetamol $1000 \mathrm{mg}$, paracetamol $500 \mathrm{mg}$ and ibuprofen $600 \mathrm{mg}$. Other medication doses and patient profiles could be adjusted in future works to provide accurate protocols for a wide range of patients based on their weights, ages and pathologies.

Another aspect to highlight is that the Intelligent Medication Controller is not focused on diagnosing the cause of the fever, so that its has been designed to recommend taking medication to patients at home previous medical prescription and medical diagnosis.

\subsection{Adjusting the medication efficacy by human expert knowledge}

The aim of the first survey was to collect the human expert knowledge to set the values of parameters for the medication efficacy model as detailed in Section 3.2: maximal efficacy degree of response $V^{*}\left(m_{d}, s\right)$ and duration of efficacy $T^{*}\left(m_{d}, s\right)$. This survey integrated two straightforward questions for each medication based on expert experience evaluated on a scale of 10 points:

- What is the value of maximum efficacy to reduce fever in patients?

- How long does it maintain its efficacy against high fever from the intake (in hours)?

Once the experts completed the form, the averaged value of effectiveness for each medication is scaled and rounded to $[0,1]$, obtaining the maximal efficacy degree $V^{*}\left(m_{d}, s\right)$. Then, the values of duration of efficacy were covered by trapezoidal membership functions $\mu_{T^{*}\left(m_{d}, s, \Delta t_{i}\right)}$ (see Eq. (2)) to define the temporal term duration of medication efficacy. The trapezoidal membership functions defined to cover the times described by the experts are shown in Table 2.

\subsection{Evaluation of fever cases and medication intakes}

The second survey presented 10 cases of fever and previous medications to evaluate the intake of antipyretics for a healthy adult whose weight is $80 \mathrm{~kg}$. The form integrated episodes of high, non presence, normal fever, disappearance and emergence of fever, as shown in Table 3. In the first three cases, the experts define the value of fever in a quantitative scale of 10 points. In the seven remaining cases, the human evaluation is more complex and the experts analyzed different 
possibilities of medication intakes based on the body temperature from the wearable device and previous intakes provided by the medication dispenser. For that, experts assigned the quantitative value of adequacy for each possibility of medication using qualitative data in the ordered scale $\{0=$ unacceptable, $1=$ slightly unacceptable, $2=$ acceptable, $3=$ perfectly acceptable $\}$. The possibilities of medication for each case were: Ibuprofen $600 \mathrm{mg}$, Paracetamol $500 \mathrm{mg}$, Paracetamol $1000 \mathrm{mg}$, Ibuprofen $600 \mathrm{mg}$ together with Paracetamol $500 \mathrm{mg}$ and not taking medication.

Once the survey was completed, we calculated a reference solution to compare the decision making of the Intelligent Medication Controller versus the human knowledge. The reference solution corresponded to the average values of fever for the first three cases and the most selected values from the ordered scale for the seven last cases. Before discussing the results of the case study, we present the adjusted parameters of the Intelligent Medication Controller:

- Bioavailability functions were obtained from literature [22,23].

- Future temporal window, for evaluating the medication gain in 12 hours $\left[t^{*}, t^{*}+12 h\right]$ because is the domain [0h, 12h] of the bioavailability functions of paracetamol and ibuprofen.

- Minimal time between two consecutive intakes, defined by the medications directions for use:

$$
\begin{aligned}
& m_{\text {ibuprofen(600mg) }}\left[t_{0}^{-}, t_{n}^{-}\right]=[4 h, 6 h], \\
& m_{\text {ibuprofen(500mg) }}\left[t_{0}^{-}, t_{n}^{-}\right]=[3 h, 4 h], \\
& m_{\text {ibuprofen }(100 m g)}\left[t_{0}^{-}, t_{n}^{-}\right]=[6 h, 8 h] .
\end{aligned}
$$

- Similarity, the difference between the degrees of medication efficacy and the degree of symptoms $s\left(t_{0}\right)-e(m, s, t)($ see Eq. (7)) is defined by a membership function where the more similar degree (differences closer to 0 ), the more degree of similarity: $\left\|s\left(t_{0}\right)-e(m, s, t)\right\|=T S\left(\left|s\left(t_{0}\right)-e(m, s, t)\right|\right)[0,0,0.25,1]$.

- Aggregation of adherence degrees. We apply the fuzzy averaging operator for the adherence degrees in the future temporal window: $a\left(m, s, t^{*}\right)=\frac{1}{\left|t_{0}, t_{f}\right|} \sum_{t}^{\left[t_{0}, t_{f}\right]}\left\|s\left(t^{*}\right)-e(m, s, t)\right\|$

- Aggregation of contraindication degrees. We apply the maximal fuzzy operator for aggregating the contraindication degrees in the future temporal window in Eq. (8): $c\left(m, s, t^{*}\right)=\max _{t}^{\left[t_{0}, t_{f}\right]} c(m, s, t)$. Note that this semantic is implemented to avoid drastically medication intakes which overcome the limits defined by their directions of use.

- Evaluation of gain. In the Eq. (9), the product operator has been defined as the t-norm between adherence and contraindication degrees : $g\left(m, s, t^{*}\right)=a\left(m, s, t^{*}\right) \times 1-c\left(m, s, t^{*}\right)$.

- Defuzzification degree of gain. The degree of gain $g\left(m, s, t_{0}\right)$ is translated into the qualitative values of the ordered scale $\{0=$ unacceptable, $1=$ slightly unacceptable, $2=$ acceptable, $3=$ perfectly acceptable $\}$ using a case function:

$$
D\left(g\left(m, s, t_{0}\right)\right)\left[l_{0}, l_{1}, l_{2}\right]= \begin{cases}\text { unacceptable } & g\left(m, s, t_{0}\right) \leq l_{0} \\ \text { slightly unacceptable } & l_{0} \leq g\left(m, s, t_{0}\right) \leq l_{1} \\ \text { acceptable } & l_{1} \leq g\left(m, s, t_{0}\right) \leq l_{2} \\ \text { perfectly acceptable } & l_{2} \leq g\left(m, s, t_{0}\right)\end{cases}
$$

The values of the interval functions have been booted until obtaining the best performance for $l_{0}=0.04, l_{1}=0.3, l_{2}=0.9$.

In Table 4, we show the reference solution from human expert versus the solutions from the Intelligent Medication Controller. In order to compute the accuracy, we have compared the values from the order scales by normalizing the difference to a percentage: $\frac{|s 1-s 2| * 100}{3}$. First, we highlight the global accuracy obtained $(\mathbf{9 1 . 3 \%})$, which represents an encouraged adherence to human decision making with a flexible analysis of complex episodes. In addition, the main differences consist of not taking medication because the human experts propose medications in a wider time range than the Intelligent Medication Controller. This is due to the fact that Intelligent Medication Controller implements a continuous real time analysis which enables waiting until optimizing the medication intakes to the fever degree. For example, the recommendation of no taking medication in case $s_{5}$ changes from 3 at 22.00 h to the value of the reference solution 2 at 22.15 h. If we take into account the proposed doses of medication we obtain an accuracy of $\mathbf{9 5 . 2 \%}$. Also, it is noteworthy that our system reached $\mathbf{1 0 0 \%}$ on the key values of contraindications which provides a secure response regarding the limits set in the medication directions of use.

\section{Conclusions}

In this paper, we have proposed an Intelligent Medication Controller which analyzes data streams from wearable body temperature devices and expends adequate medication in a remote dispenser installed at home. The system aims at providing accuracy and adherence to user fever in the decision making of medication intakes in order to adjust the doses and waiting time between intakes.

The pharmacokinetic and pharmacodynamic analysis using expert knowledge under fuzzy linguistic has been driven effectively to calculate the bioavailability, efficacy and contraindications. A case study with a complete range of fever episodes is presented to compare the decision making in medication intakes of antipyretic between human expert and the Intelligent Medication Controller. We have obtained a promising result of similarity regarding expert decision keeping a flexible analysis and assuring a secure response for contraindication cases. 
Table 4

Reference human decision (RHD) versus the Intelligent Medication Controller (IMD).

\begin{tabular}{|c|c|c|c|}
\hline Case & Fever-medication & RHD & IMD \\
\hline$s_{1}$ & fever & 1.25 & 1.3 \\
\hline$s_{2}$ & fever & 9.6 & 9.6 \\
\hline$s_{3}$ & fever & 7.25 & 7.2 \\
\hline$s_{4}$ & $i 600$ & 3 & 3 \\
\hline$s_{4}$ & $p 500$ & 1 & 1 \\
\hline$s_{4}$ & p1000 & 0 & 0 \\
\hline$s_{4}$ & $i 600+p 500$ & 1 & 1 \\
\hline$s_{4}$ & 0 & 0 & 1 \\
\hline$s_{5}$ & $i 600$ & 0 & 0 \\
\hline$s_{5}$ & p500 & 3 & 2 \\
\hline$s_{5}$ & p1000 & 2 & 2 \\
\hline$s_{5}$ & $i 600+p 500$ & 0 & 0 \\
\hline$s_{5}$ & 0 & 2 & 3 \\
\hline$s_{6}$ & $i 600$ & 2 & 2 \\
\hline$s_{6}$ & p500 & 2 & 2 \\
\hline$s_{6}$ & p1000 & 3 & 3 \\
\hline$s_{6}$ & $i 600+p 500$ & 2 & 2 \\
\hline$s_{6}$ & 0 & 0 & 0 \\
\hline$s_{7}$ & $i 600$ & 0 & 0 \\
\hline$s_{7}$ & p500 & 3 & 2 \\
\hline$s_{7}$ & p1000 & 2 & 2 \\
\hline$s_{7}$ & $i 600+p 500$ & 0 & 0 \\
\hline$s_{7}$ & 0 & 2 & 3 \\
\hline$s_{8}$ & $i 600$ & 0 & 0 \\
\hline$s_{8}$ & p500 & 3 & 3 \\
\hline$s_{8}$ & p1000 & 3 & 3 \\
\hline$s_{8}$ & $i 600+p 500$ & 0 & 0 \\
\hline$s_{8}$ & 0 & 1 & 2 \\
\hline$s_{9}$ & $i 600$ & 2 & 2 \\
\hline$s_{9}$ & p500 & 3 & 2 \\
\hline$s_{9}$ & p1000 & 2 & 2 \\
\hline$s_{9}$ & $i 600+p 500$ & 0 & 1 \\
\hline$s_{9}$ & 0 & 2 & 3 \\
\hline$s_{10}$ & $i 600$ & 2 & 2 \\
\hline$s_{10}$ & p500 & 2 & 2 \\
\hline$s_{10}$ & p1000 & 3 & 3 \\
\hline$s_{10}$ & $i 600+p 500$ & 2 & 2 \\
\hline$s_{10}$ & 0 & 1 & 1 \\
\hline Accuracy & \multicolumn{2}{|l|}{ Total } & $91.3 \%$ \\
\hline Accuracy & \multirow{2}{*}{\multicolumn{2}{|c|}{ Medication+Fever }} & $95.2 \%$ \\
\hline Accuracy & & & $100.0 \%$ \\
\hline
\end{tabular}

In addition, we have completed the Intelligent Medication Controller with a prototype of smart object at home: a remote medication dispenser with a 3D printer design which implements a remote communication by IoT.

In future works, we will put our efforts in integrating accurate protocols for specific patient profiles based on their weights, ages and pathologies. It will involve an adjustement of parameters by expert knowledge and other comprehensive studies of adherence in the decision making of medication intakes. Furthemore, we will extend the approach to include prospective analysis for estimating which is the best medication option in a future time and notifying and reminding it to patients by means of wearable devices.

Despite the fact that the work is focused on the evaluation of fever, we highlight that the methodology has been defined in terms of adherence to a sympton, enabling future extensions to manage other common pathologies such as chronic pain, which involves controlling the medication on demand [28]. Moreover, we hope collaborating with Health Centers in order to evaluate the Intelligent Medication Controller in groups of patients with one of these particular daily diseases.

\section{Acknowledgement}

This contribution has been supported by research projects: AC20/2015-10 and PI-0203-2016.

\section{References}

[1] Atzori L, Iera A, Morabito G. The internet of things: a survey. Comput Netw 2010;54(15):2787-805. Kortuem, G., Kawsar, F., Sundramoorthy, V., \& Fitton, D. (2010). Smart objects as building blocks for the internet of things. IEEE Internet Computing, 14(1), 44-51.

[2] Xu H, Collinge WO, Schaefer LA, Landis AE, Bilec MM, Jones AK. Towards a commodity solution for the internet of things. Comput Electr Eng 2016;52:138-56 
[3] Weiser M, Perera C, Zaslavsky A, Christen P, Georgakopoulos D. The computer for the 21st century. Sci Am 1991;265(3):94-104. Varshney, U. (2007). Pervasive healthcare and wireless health monitoring. Mobile Networks and Applications, 12(2-3), 113-127. 2014,Context aware computing for the Internet of Things: A survey, IEEE Communications Surveys \& Tutorials, 16, 1, 414-454.

[4] Alam MM, Hamida EB. Surveying wearable human assistive technology for life and safety critical applications: standards, challenges and opportunities. Sensors 2014;14(5):9153-209.

[5] Ashman JJ. Multiple chronic conditions among US adults who visited physician offices: data from the national ambulatory medical care survey, 2009. Prevent Chronic Disease; 2013. p. 10.

[6] Veazie PJ. An individual-based framework for the study of medical error. Int J Qual Health Care 2006;18(4):314-19.

[7] Classen DC, Pestotnik SL, Evans RS, Lloyd JF, Burke JP. Adverse drug events in hospitalized patients: excess length of stay, extra costs, and attributable mortality. JAMA 1997;277(4):301-6.

[8] Barat I, Andreasen F, Damsgaard EMS. The consumption of drugs by 75-year-old individuals living in their own homes. Eur J Clin Pharmacol 2000;56(6-7):501-9.

[9] Tsai PH, Yu CY, Wang WY, Zao JK, Yeh HC, Shih CS, et al. IMAT: intelligent medication administration tools. Proc IEEE Healthcom 2010. (July 2010).

[10] Suzuki T, Nakauchi Y. A remote monitoring system of medication compliance using distributed RFID readers in an intelligent living environment. Adv Biomed Eng 2014;3(0):106-15

[11] Jara AJ, Zamora MA, Skarmeta AF. Drug identification and interaction checker based on iot to minimize adverse drug reactions and improve drug compliance. Pers Ubiquitous Comput 2014;18(1):5-17

[12] Zadeh LA. Fuzzy sets. Inf Control 1965;8(3):338-53.

[13] Medina J, Espinilla M, Martínez L. Approach for subscribing to fuzzy temporal aggregation of heterogeneous sensor streams in real-time distributed environments. Int J Commun Syst 2016.

[14] Patel MS, Asch DA, Volpp KG. Wearable devices as facilitators, not drivers, of health behavior change. JAMA 2015;313(5):459-60.

[15] Hamine S, Gerth-Guyette E, Faulx D, Green BB, Ginsburg AS. Impact of mhealth chronic disease management on treatment adherence and patient outcomes: a systematic review. J Med Internet Res 2015;17(2):e52.

[16] Shargel L, Wu-Pong S, Yu AB. Applied biopharmaceutics \& pharmacokinetics. McGraw-Hill; 2007

[17] Lees P, Cunningham FM, Elliott J. Principles of pharmacodynamics and their applications in veterinary pharmacology. J Vet Pharmacol Ther 2004;27(6):397-414. doi:10.1111/j.1365-2885.2004.00620.x. PMID 15601436.

[18] Neubig R, Spedding M, Kenakin T, Christopoulos A. International union of pharmacology committee on receptor nomenclature and drug, classification (december 2003). international union of pharmacology committee on receptor nomenclature and drug classification. XXXVIII. update on terms and symbols in quantitative pharmacology. Pharmacol Rev 2003;55(4):597-606. doi:10.1124/pr.55.4.4. PMID 14657418.

[19] Welling PG. Differences between pharmacokinetics and toxicokinetics. Toxicol Pathol 1995;23(2):143-7.

[20] Medina J, Espinilla M, Nugent C. Real-time fuzzy linguistic analysis of anomalies from medical monitoring devices on data streams. Fut Pervas Health Worksh 2016. doi:10.4108/eai.16-5-2016.2263877.

[21] RP S. A modification of receptor theory. Br J Pharmacol Chemother 1956;11(4):379-93 doi:10.1111/j.1476-5381.1956.tb00006.x. PMC 1510558free to read. PMID 13383117.

[22] Farre M, Roset PN, Abanades S, Menoyo E, Alvarez Y, Rovira M, et al. Study of paracetamol 1-g oral solution bioavailability. Methods Find Exp Clin Pharmacol 2008;30(1):37-42.

[23] Dewland PM, Reader S, Berry P. Bioavailability of ibuprofen following oral administration of standard ibuprofen, sodium ibuprofen or ibuprofen acid incorporating poloxamer in healthy volunteers. BMC Pharmacol Toxicol 2009;9(1):19.

[24] Bobbink R, Hettelingh JP. Review and revision of empirical critical loads and dose-response relationships, 2325. Proceedings of An Expert Workshop, Noordwijkerhout; 2010.

[25] Kenakin T. A pharmacology primer: theory, application and methods. Academic Press; 2009.

[26] Carruthers SG. Duration of drug action. Am Fam Physician 1980;21(2):119-26.

[27] Glockner I, Knoll A. Fuzzy quantifiers: a natural language technique for data fusion. In: Proceedings of the fourth international conference on information fusion (Fusion 2001); 2001.

[28] Fairbank JC, Couper J, Davies JB, O’brien JP. The oswestry low back pain disability questionnaire. Physiotherapy 1980;66(8):271-3.

[29] Gordon M. Manual of nursing diagnosis. Jones \& Bartlett Publishers; 2014.

[30] Pantelopoulos A, Bourbakis NG. A survey on wearable sensor-based systems for health monitoring and prognosis. IEEE Trans Syst Man Cybern Part C (Appl Rev) 2010;40(1):1-12. 
Javier Medina received the M.Sc. and Ph.D. degrees in Computer Science in the University of Granada, Spain, in 2007 and 2010 , respectively. He is working as researcher in the University of Granada and University of Jaén. His research interests encompass fuzzy logic, intelligent systems, ubiquitous computing and ambient intelligence.

Macarena Espinilla received the M.Sc. and Ph.D. degrees, both in Computer Science, from the University of Jaén (Jaén, Spain), in 2006 and 2009, respectively. She is currently Associate Professor in the Department of Computer Systems at University of Jaén. Her current research interests include smart environments, ubiquitous computing, ambient assisted living and ambient intelligence.

Ángel-Luis García-Fernández is a lecturer at the Computer Science Department of the University of Jaén (Spain) since 2004. He earned his Ph.D. in Computer Science in 2007, and his research topics include Computer Graphics (Solid Modeling and 3D printing), Geomatics, Information Systems, Virtual Archaeology and more recently, Smart Environments

Luis Martínez received M.Sc. and Ph.D. degrees in Computer Sciences from the University of Granada, Spain, in 1993 and 1999 , respectively. He is Full Professor at the University of Jaén and Co-Editor in Chief of the International Journal of Computational Intelligence Systems. His research interests are fuzzy decision making, ambient intelligence and recommender systems. He received twice the IEEE TFS Outstanding Paper Award 2008 and 2012. 